\title{
Characterization of Mn oxides using "flank" method in SEM-SXES system
}

\author{
Yohei Kojima ${ }^{1}$ and Natasha Erdman ${ }^{2}$
}

${ }^{1}$ JEOL, Ltd., Akishima, Tokyo, Japan, ${ }^{2}$ JEOL USA Inc, Peabody, Massachusetts, United States

A soft X-ray emission spectrometer (SXES) is a recently developed spectrometer with high energy resolution and high sensitivity that can be installed in SEM and EPMA (Electron Probe Micro Analyzer) [1]. FE-SEM equipped with SXES enables analysis of the chemical state of micro- or even nano-textures. This paper presents analysis of valence state in Mn oxides using the SEM-SXES system. Mn-containing oxides are widely used in battery cathode materials and perovskite-type magnetic materials. The changes in the valence of Mn affect their physical properties and performance. Therefore, characterization of Mn valence state in Mn-containing oxides is very important. The SXES examination was performed by using a Schottky type FE-SEM (JEOL JSM-IT800 (SHL)) equipped with a SXES (JEOL SS-94040SXSER). The energy range of this spectrometer with a JS2000 diffraction grating is from 350 to $2300 \mathrm{eV}$, and the energy resolution is defined to be $5.0 \mathrm{eV}$ by the FWHM of Fe-L $\alpha$ line. MnO, Mn2O3 and MnO2 purchased from Hori Mineralogy were used for samples. Figure 1 shows the Mn-L emission spectra of MnO, Mn2O3 and $\mathrm{MnO} 2$ collected by SXES at accelerating voltages of 2, 5 and $10 \mathrm{kV}$ and self-absorption spectra calculated from the SXES spectra. The peak position of the Mn-L $\alpha$ line shifts to the lower energy with increasing accelerating voltage and the intensity ratios of $\mathrm{Mn} L \beta / \mathrm{L} \alpha$ do not exhibit any tendency. This indicates that the conventional peak-top intensity ratio and peak-area intensity ratio methods cannot be applied. This is due to self-absorption effect of characteristic X-rays. Characteristic X-rays generated by electron beam irradiation are affected by self-absorption in which energy is absorbed by the sample itself before the X-rays escape from the sample surface. Generally, the self-absorption effect becomes more prominent with increasing accelerating voltage. Therefore, the self-absorption spectra can be expressed by dividing the SXES spectrum at a low accelerating voltage by the one at a high accelerating voltage (Fig. 1) [2]. The energy position of the strongest self-absorption is constant regardless of the accelerating voltage (Fig. 1), and such phenomenon can be also observed in the case of Fe-L emission [2]. Hofër et al. has developed a method called the "flank" method which takes advantage of the difference in chemical bonding and the self-absorption effect due to the difference in the Fe valence [2]. This method follows the steps below: 1) Measure the spectra of the end-member of the material and calculate the difference spectrum from them. 2) Calculate the intensity ratio of $L \beta$ and $L \alpha$ at the flank positions which are determined by the maximum or minimum energy position in the L $\alpha$ and L $\beta$ regions of the difference spectrum. 3) Plot the obtained $L \beta / L \alpha$ intensity ratio related to the valence obtaining a calibration curve. They reported that the "flank" method has a higher sensitivity to Fe valence than the peak-top intensity ratio and peak-area intensity ratio methods in Fe oxides and garnet system [2,3]. In this study, we clarified whether this method can be applied to the analysis of Mn valence change. Figure 2a shows the SXES spectra of $\mathrm{MnO}, \mathrm{Mn} 2 \mathrm{O} 3$ and $\mathrm{MnO} 2$ taken at an accelerating voltage of $10 \mathrm{kV}$ and the difference spectra with respect to $\mathrm{MnO} 2$. The flank positions in $\mathrm{L} \alpha$ and $\mathrm{L} \beta$ regions are respectively determined to be 639 and $647 \mathrm{eV}$ by the difference spectra. The intensity ratio of $\mathrm{Mn} \mathrm{L} \beta / \mathrm{L} \alpha$ are calculated at the flank positions. The intensity ratios obtained by the "flank" method and two conventional methods are plotted as a function of Mn valence in Fig. 2b. The "flank" method has a higher resolution and its calibration curve tends to be more linear compared to the two conventional methods. This trend is observed at an accelerating voltage of $5 \mathrm{kV}$. The results in this study indicate that the "flank" method is applicable to Mn oxides as well as Fe oxides. In the "flank" method, a calibration curve should be obtained for each system because the selfabsorption effect changes when the system becomes multi-component. Since Mn-containing oxides, which are widely used for industrial applications such as a cathode of LIBs, are multi-component, we would like to establish this method and apply it to such materials in the future. 

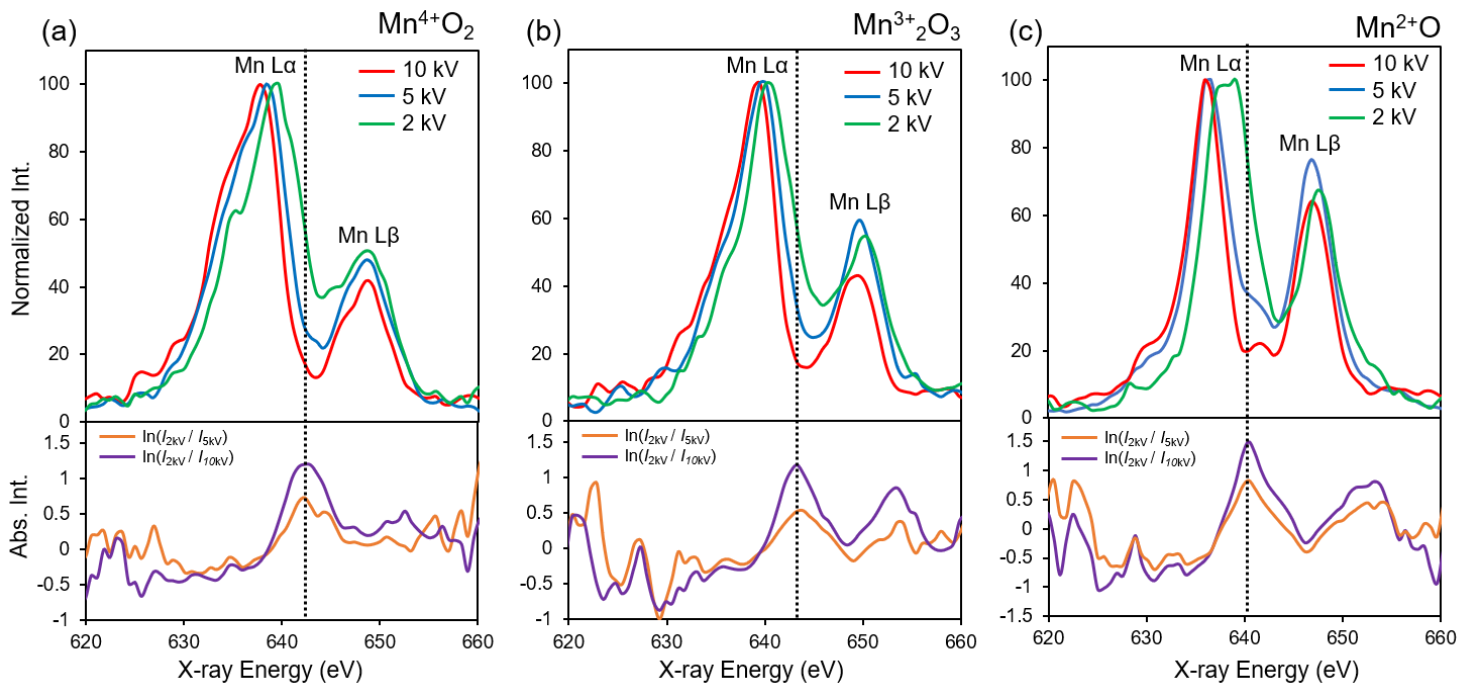

Figure 1. SXES (upper graphs) and self-absorption spectra (lower graphs) of (a) $\mathrm{MnO} 2$, (b) $\mathrm{Mn} 2 \mathrm{O} 3$ and (c) $\mathrm{MnO}$.

(a)

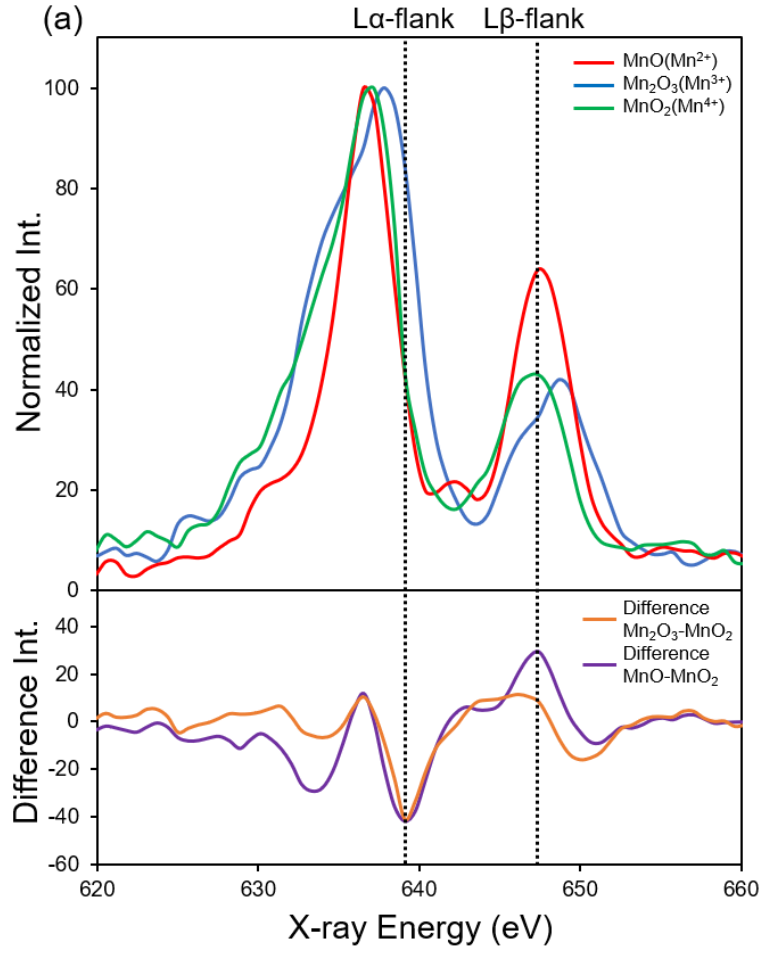

(b)

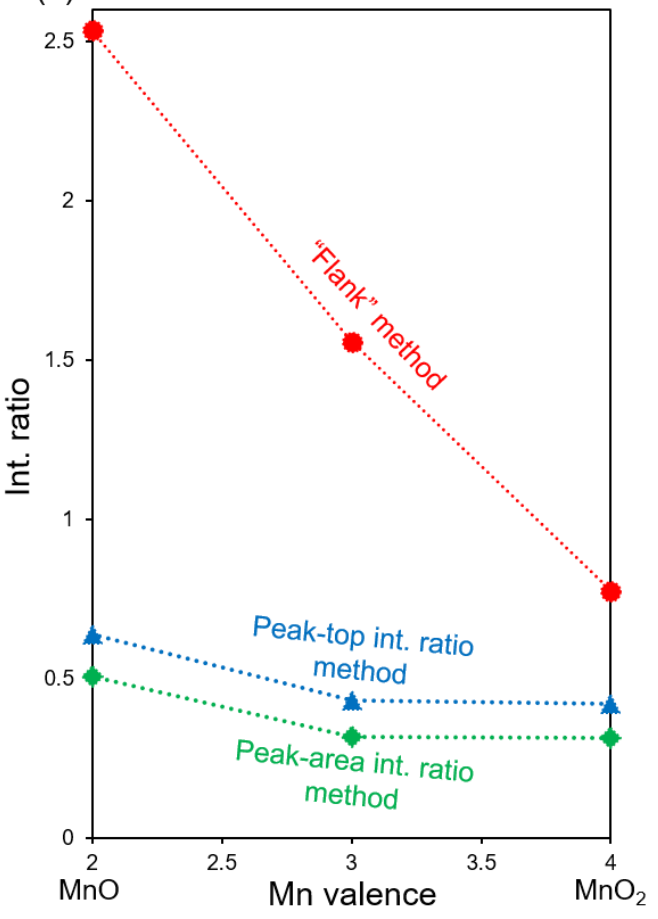

Figure 2. (a) SXES spectra (upper graph) of $\mathrm{MnO}, \mathrm{Mn} 2 \mathrm{O} 3$ and $\mathrm{MnO}$, and difference spectra (lower graph) with respect to $\mathrm{MnO} 2$ at acceleration voltage of $10 \mathrm{kV}$, (b) the intensity ratio plotted as a function of Mn valence by each method.

\section{References}

[1] H. Takahashi et al., IOP Conf. Ser.: Mater. Sci. Eng., (2016) 109012017

[2] H. Hofër et al., Eur. J. Mineral., (1994) 6 407-418.

[3] H. Hofër and G. P. Brey, Am. Mineral., (2007) 92 873-885. 